\title{
IL-17 disrupts corneal barrier following desiccating stress
}

\author{
CS De Paiva ${ }^{1}$, S Chotikavanich ${ }^{1}$, SB Pangelinan ${ }^{1}$, JD PitcherIII ${ }^{1}$, B Fang ${ }^{1}$, X Zheng $^{1}$, P Ma $^{1}$, WJ Farley ${ }^{1}$, \\ KF Siemasko ${ }^{2}$, JY Niederkorn ${ }^{3}$, ME Stern ${ }^{2}$, D-Q Li ${ }^{1}$ and SC Pflugfelder ${ }^{1}$
}

Thelper (Th)-17 is a recently identified subtype of Th response that has been implicated in host defense and autoimmunity. We investigated whether there is evidence for a Th-17 response in human and experimental murine dry eye (DE). Gene expression in the human DE conjunctiva showed increased levels of the Th-17 inducers, interleukin (IL)-23, IL-17A, and interferon-gamma (IFN- $\gamma$ ). In the murine model, we found that desiccating stress increased matrix metalloproteinase-9, Th-17-associated genes (IL-6, IL-23, transforming growth factor- $\beta 1$ and -2, IL-23R, IL-17R, IL-17A, retinoid-related orphan receptor- $\gamma \mathrm{t}$, and CC chemokine attractant ligand-20) and IFN- $\gamma$ in cornea and conjunctiva. Furthermore, we found a significantly increased concentration of IL-17 in tears and number of IL-17producing cells on the ocular surface. Antibody neutralization of IL-17 ameliorated experimental DE-induced corneal epithelial barrier dysfunction and decreased the expression of matrix metalloproteinases 3 and 9 . Taken together, these findings suggest that IL-17 has a role in corneal epithelial barrier disruption in DE.

\section{INTRODUCTION}

Thelper (Th)-17 is a newly recognized CD4 + T-cell subset that is distinct from traditional Th- 1 and Th-2 lineages. Th- 1 responses are important for controlling viral, fungal, and intracellular bacterial infections. Th- 1 cells are classically identified by the production of interferon-gamma (IFN- $\gamma$ ). Once committed, the Th-1 cells activate macrophages and induce IgG2a production by $\mathrm{B}$ cells. Th-2 responses are frequently found in allergic diseases, such as asthma, and are particularly important in the host response to parasites and helminthes in the gut. Th-2-cell differentiation is promoted by interleukin (IL)- 4 and it is characterized by the production of IL-4, IL-13, and IL-5. The Th-2-committed cells promote IgG1 and IgE class switching and eosinophil recruitment. ${ }^{1}$

Interleukin-17A is the "signature" cytokine of the Th-17 pathway. Th-17-committed cells can produce IL-17A, IL-21, IL-22, CC chemokine attractant ligand-20 (CCL20), and IL-17F.,3 IL-17A has also been shown to promote production of IL-1, tumor necrosis factor alpha (TNF- $\alpha$ ), IL-6, IL-8, and matrix metalloproteinases (MMPs) by epithelial cells and fibroblasts. ${ }^{4-6}$ Th-17 cells are important in responding to extracellular bacterial and fungal pathogens, by recruiting neutrophils and macrophages to infected tissues. ${ }^{7-9}$
Studies in mice have found that Th-17 differentiation is driven by IL- 6 and transforming growth factor beta (TGF- $\beta)^{10-12}$ and requires the lineage-specific transcript factor, retinoid-related orphan receptor- $\gamma \mathrm{t}(\mathrm{ROR} \gamma \mathrm{t}) .{ }^{13} \mathrm{IL}-23$ has been implicated in the survival and proliferation of Th-17 cells. ${ }^{14,15}$ In vitro, IL-1 $\beta$ and TNF- $\alpha$ can amplify differentiation of IL-17 + -producing cells. ${ }^{10}$ In humans, Th-17 polarization is induced by IL- $1 \beta$ and enhanced by IL- 6 and IL-23. ${ }^{16,17}$

The biological role of Th-17 cells is not fully understood; however, IL-17 has been implicated in autoimmunity in humans and also in animal models, such as experimental autoimmune encephalomyelitis, autoimmune uveitis, collagen-induced arthritis, and inflammatory bowel disease. ${ }^{18-21}$ IL-17 has been found to be elevated in psoriasis lesions, ${ }^{22,23}$ in the synovial fluids of patients with rheumatoid arthritis, ${ }^{24}$ and in vitreous of patients with uveitis. ${ }^{25,26}$

Dry eye is the second most common problem of patients seeking eye care, and is characterized by eye irritation symptoms and blurred vision. The prevalence of dry eye (DE) increases with age, ${ }^{27}$ with a prevalence of $6 \%$ at 40 years of age, increasing to $15-25 \%$ in the population over the age of 65 years. ${ }^{28,29}$ Among DE patients, 11\% have been estimated to have the

${ }^{1}$ Department of Ophthalmology, Ocular Surface Center, Cullen Eye Institute, Baylor College of Medicine, Houston, Texas, USA. ²Department of Biological Sciences,

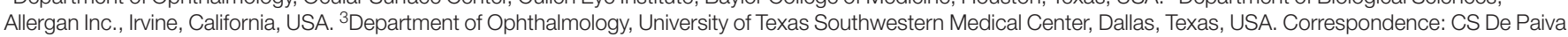
(cintiadp@bcm.tmc.edu)

Received 14 September 2008; accepted 21 December 2008; published online 25 February 2009. doi:10.1038/mi.2009.5 
systemic autoimmune condition Sjögren's syndrome, a severe and potentially blinding condition. ${ }^{30}$

Dry eye can have a major impact on quality of life. The presence of DE impacts functional vision and the ability to perform daily activities such as reading, computer use, and driving. ${ }^{31}$ Disrupted corneal epithelial barrier and accelerated corneal epithelial loss in DE lead to corneal surface irregularity, which degrades visual function by decreasing contrast sensitivity and functional visual acuity.

We found earlier that disruption of corneal barrier function in $\mathrm{DE}$ is mediated by increased production of MMPs, particularly, MMP-9. The mechanisms that stimulate increased protease production and activation have not been fully elucidated, but proinflammatory cytokines and chemokines, such as IL- 1 and TNF- $\alpha$, which increase in the tears in DE disease, stimulate MMP production by the corneal epithelium. ${ }^{32,33}$ Furthermore, inflammation in the lacrimal glands, cornea, and conjunctiva, which results in decreased tear production and conjunctival goblet cell loss, has been induced by transferring CD4 $+\mathrm{T}$ cells from mice subjected to experimental DE to T-cell-deficient nude mice that have not been exposed to desiccating stress. ${ }^{34}$ These findings suggest that $\mathrm{CD} 4+\mathrm{T}$ cells may be involved in the pathogenesis of DE.

The goal of our study was twofold, first, to determine if the desiccating stress of DE induces a Th-17 inflammatory skewed response on the ocular surface, and second, to determine if the IL-17 is implicated in the corneal epithelial disease in $\mathrm{DE}$. This was accomplished by evaluating the expression of Th-17 promoters and IL-17 in human DE patients and in a murine DE model. Furthermore, the effect of IL-17 neutralization on corneal epithelial barrier function was evaluated in an experimental DE model.

\section{RESULTS}

\section{Evidence for Th-17 inflammation in the conjunctiva of DE patients}

Chronic inflammation has been detected in the conjunctiva of patients with deficiency/dysfunction of the tear film, commonly called DE. Compared with normal eyes, significantly increased levels of IL- $1 \alpha$, IL-6, IL-8, TNF- $\alpha$, and TGF- $\beta 1$ RNA transcripts have been found in the conjunctival epithelium of patients with Sjögren's syndrome, the most severe form of $\mathrm{DE} .{ }^{32}$

To determine if Th-17 pathways are involved in DE pathogenesis, we measured levels of Th-17-associated cytokines in human conjunctival epithelium obtained by impression cytology in 17 patients with newly diagnosed $\mathrm{DE}$ ranging in severity from level 1 to 4 [level $1(n=2)$, level $2(n=2)$, level $3(n=4)$, and level 4 $(n=9)]$ and 17 normal subjects, with no evidence of DE, using real-time PCR. The demographic features of patients and control subjects are shown in Table 1. Levels of mRNA transcripts encoding MMP-9 and Th-17 (IL-6, IL-23, IL-17A, TGF- $\beta 1$, TGF- $\beta 2$, and IL-15), Th- 1 (IFN- $\gamma$, IL-2, and IL-12), and Th-2 (IL-4 and IL-13) cytokine families in the conjunctival epithelia are shown in Figure 1. Significantly higher levels of MMP-9, TNF- $\alpha$, IL- $1 \beta$, IL-6, TGF- $\beta 2$, IL-23, IL- 17 , and IFN- $\gamma$ transcripts were observed in DE patients than normal subjects. There was no difference in levels of IL-2, IL-12, and IL-15 transcripts. IL-13 was detected in only 4 out of 17 normal subjects and 3 out of 17
Table 1 Demographic features of normal and dry eye subjects

\begin{tabular}{lcc}
\hline Mean age (years) & $\begin{array}{c}\text { Normal subjects } \\
\mathbf{3 5 . 4} \text { (range 24-61) }\end{array}$ & $\begin{array}{c}\text { Dry eye subjects } \\
\mathbf{5 7 . 0} \text { (range } \mathbf{3 4 - 8 1})\end{array}$ \\
\hline Gender & & \\
Female & $10 / 17$ & $15 / 17$ \\
Male & $7 / 17$ & $2 / 17$ \\
\hline
\end{tabular}

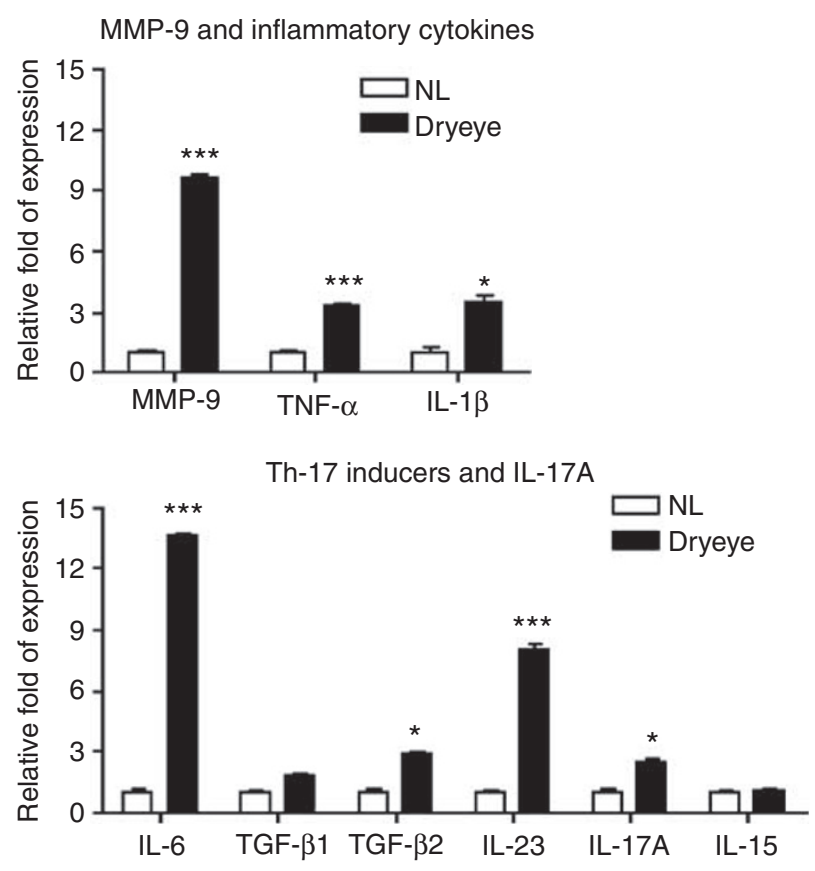

Th-1 and -2 cytokines

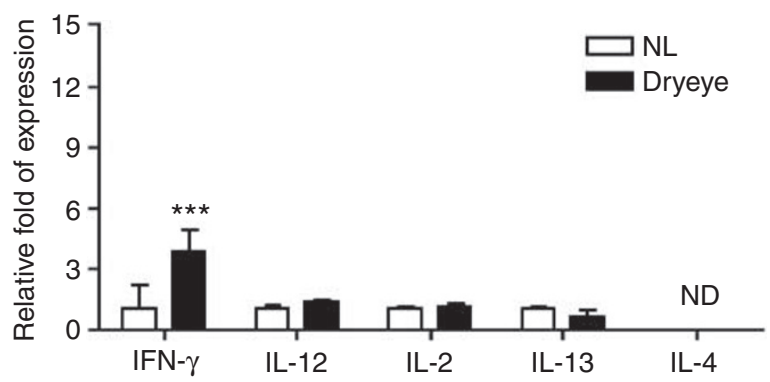

Figure $1 \mathrm{mRNA}$ transcripts in human conjunctival epithelia from normal subjects and patients with $\mathrm{DE}(n=17) .{ }^{\star} P<0.05,{ }^{\star \star \star} P<0.001, \mathrm{ND}=$ nondetectable. DE, dry eye.

patients, whereas IL-4 transcripts were undetectable in all samples obtained from both groups, although they were detected in samples obtained from two patients with atopic conjunctivitis (data not shown). We also performed an analysis correlating levels of IL-6, IL-23, and TGF- $\beta 1$ mRNA with the levels of clinical severity. We found that there was a significant and direct correlation between the levels of expression of IL-6, IL-23, and TGF- $\beta 1$ $\left(R^{2}\right.$ (coefficient of correlation) $=0.3701, R^{2}=0.3139, R^{2}=0.3538$; $P=0.0001, P=0.005$, and $P=0.0003$, respectively) with clinical severity (Supplementary Figure 1). 
These findings indicate that DE elicits a mixed Th-17 and Th-1 response in the human conjunctival epithelia.

\section{Desiccating stress induces a Th-17 response in the murine cornea and conjunctiva}

On the basis of these findings in conjunctiva obtained from human DE patients, we decided to further evaluate the expression of these genes on the ocular surface and in the draining lymph nodes of mice subjected to desiccating stress for 5 or 10 days (DS5 and DS10, respectively), using an established experimental murine DE model. ${ }^{34-37}$ Non-stressed (NS) mice served as controls.

We have observed earlier that mice subjected to desiccating stress develop a CD $4+$ T-cell infiltration in the goblet-cell-rich area of the conjunctiva. ${ }^{38}$ We have also observed occasional CD4 + T-cell infiltration of the peripheral cornea after desiccating stress (DS) (data not shown). We observed that adoptive transfer of DS CD4 + T cells into T-cell-deficient nude mice was able to produce lacrimal keratoconjunctivitis in mice that were never exposed to desiccating stress. ${ }^{34}$ To confirm our earlier finding, we performed flow cytometry on freshly isolated cells from the ocular surface (i.e., cornea and conjunctiva) and cervical lymph nodes (CLNs) that were stained for CD4. Our results show that both tissues had an increase in CD4 + T-cell population (Figure 2 and Supplementary Figure 2). We hypothesized that these findings may be associated with a Th-17 response.

We used this murine DS model to evaluate the levels of mRNA transcripts encoding MMP-9, Th-17 inducers, and IL-17-, Th-1-, and Th-2-associated genes in the cornea, conjunctiva (Table 2), and CLN (Supplementary Table 1) in DS5 or DS10 mice by real-time PCR.

In the cornea epithelia, there was a significant increase of MMP-9, TGF- $\beta 1$, TGF- $\beta 2$, IL-17A, IL-23, IFN- $\gamma$, and IL-2 (at DS5), and CCL20 (at DS5 and DS10) and IL-6 (at DS10) transcript levels compared with the NS group. Neither IL-4 nor IL-22 mRNA transcripts were found in the cornea epithelia.

Significantly higher levels of IL-23, IL-23R, IL-17R, ROR $\gamma \mathrm{t}$ (all at DS5), TGF- $\beta 1$, IL-17A, IL-6, IL-22 (both at DS5 and DS10), MMP-9, and IFN- $\gamma$ (at DS10) transcripts were observed in the conjunctiva of DS mice than the NS group. No change was observed in IL-15, TGF- $\beta 2$, IL-18, IL-18R, T-bet, CCL20, IL21 , and STAT3 levels. We found that there was a nonsignificant trend toward decreasing levels of IL-2, IL-12, IL-4, IL-13, and GATA3 transcripts after DS.

In the draining lymph node, a significant increase of TGF- $\beta 2$ (at DS5) and IL-17A (both at DS5 and DS10) was noted, whereas a nonsignificant increase of ROR $\gamma \mathrm{t}$ was observed at DS5.

Taken together, these results show that DS induces a stronger Th-17 pattern response in the cornea and conjunctiva than in the regional lymph node and prompted us to further investigate the role of Th-17 in DE.

\section{Desiccating stress upregulates Th-17 inducers on the ocular surface}

The gene expression studies were confirmed by evaluating the protein expression of Th-17 inducers, IL-6, IL-23, IL-23R, and

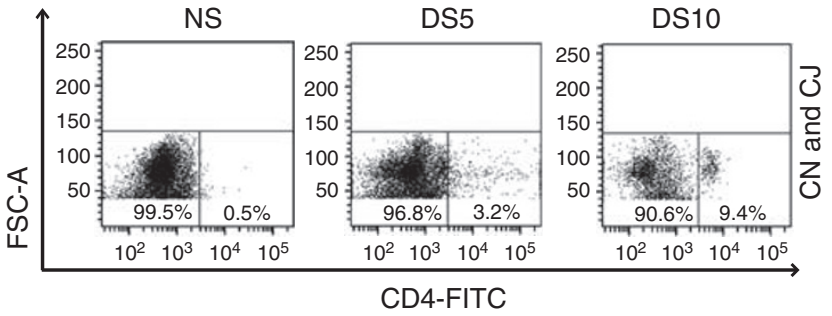

Figure 2 Flow cytometry analysis of freshly isolated cells from the cornea (CN) and conjunctiva (CJ) stained with CD4-FITC-conjugated antibody in NS control (NS), 5 days (DS5) or 10 days (DS10) of desiccating stress (DS) ( $n=5$ per group). Lymphocytes were gated based on characteristic light-scatter properties, single lymphocytes were gated based on forward scatter height vs. forward scatter area (FSC-A). Numbers in the quadrants indicate the percentage of cells.

TGF- $\beta$, by immunostaining, and by Luminex immunobead assay in corneas and conjunctivae obtained from NS, DS5, and DS10 mice. We also evaluated the concentration of IL-12p40 and IL-12p70 in tears. CCL20, a chemoattractant that was shown to be induced by IL-17, ${ }^{2}$ was also evaluated by immunofluorescent staining.

Dry eye induced a significant increase in IL-6 and IL-23 immunoreactivity in the cornea and conjunctival epithelia at DS5 and DS10 (Figure 3a-d). These results were confirmed by measuring the concentrations of these cytokines in corneal and conjunctival lysates using Luminex immunobead assay (Supplementary Figure 3a). Scattered IL-23R-positive cells were found to infiltrate the conjunctival epithelium (Figure 3e, arrows). Desiccating stress induced a significant increase in CCL20 staining in the upper layers of the corneal epithelium, especially in the peripheral cornea (Figure 3f).

Total levels of TGF- $\beta 1$ and TGF- $\beta 2$ were measured using a Luminex immunobead assay in conjunctival lysates of all groups and normalized by the total protein concentration. As shown in Figure 3g, TGF- $\beta 1$ levels increased significantly at DS10, whereas TGF- $\beta 2$ did not change, in agreement with our PCR results.

We evaluated the concentrations of IL-12p40 (the shared subunit between IL-23 and IL-12) and IL-12p70 (the exclusive subunit of IL-12) in corneal and conjunctival lysates, and tears. As there are no commercially available beads to IL-23p19 (the exclusive subunit of IL-23), we evaluated the ratio of both subunits as a measure of IL-23. Desiccating stress induced a progressive increase of IL-12p40 in tears, whereas the expression of IL-12p70 decreased; therefore, the ratio of both units increased significantly, implying an increase of IL-23 (Figure 3i). These findings were confirmed by measuring the concentrations of these cytokines in the cornea and conjunctiva, in which a significant decrease in IL-12p70 at DS10 was observed in conjunctival lysates. In cornea, there was a slight increase in IL-12p40, and this was accompanied by a decrease in IL-12p70. Thus, the same pattern of this ratio observed in tears was also observed in lysates of both ocular surface tissues (Supplementary Figure $3 \mathbf{b}-\mathbf{d})$. 
Table 2 mRNA transcript levels of Th-17 inducers, Th-17, Th-1, and Th-2 pathways in the cornea and conjunctiva in non-stressed control mice (NS) and mice subjected to desiccating stress for 5 or 10 days (DS5 or DS10, respectively)

\begin{tabular}{|c|c|c|c|c|}
\hline Gene & Location & \multicolumn{3}{|c|}{ Relative fold increase } \\
\hline \multicolumn{5}{|c|}{ MMP-9 and Th-17 inducers } \\
\hline MMP-9 & Conjunctiva & $1.00 \pm 0.38$ & $1.77 \pm 0.33$ & $2.23 \pm 0.35^{\star}$ \\
\hline IL-6 & Cornea & $1.00 \pm 0.36$ & $1.55 \pm 0.25$ & $2.51 \pm 0.57^{\star}$ \\
\hline \multirow[t]{2}{*}{ IL-23 } & Cornea & $1.00 \pm 0.49$ & $2.77 \pm 0.43^{\star *}$ & $2.24 \pm 0.51$ \\
\hline & Conjunctiva & $1.00 \pm 0.48$ & $2.59 \pm 0.50^{*}$ & $2.14 \pm 0.40$ \\
\hline \multirow[t]{2}{*}{ TGF- $\beta 1$} & Cornea & $1.00 \pm 0.29$ & $1.89 \pm 0.32^{*}$ & $0.47 \pm 0.32$ \\
\hline & Conjunctiva & $1.00 \pm 0.46$ & $2.73 \pm 0.51^{* *}$ & $2.97 \pm 0.56^{\star *}$ \\
\hline TGF- $\beta 2$ & Cornea & $1.00 \pm 0.43$ & $3.01 \pm 0.52^{*}$ & $0.49 \pm 0.26$ \\
\hline \multirow[t]{2}{*}{ IL-23R } & Cornea & $1.00 \pm 0.41$ & $1.37 \pm 0.49$ & $0.56 \pm 0.33$ \\
\hline & Conjunctiva & $1.00 \pm 0.33$ & $2.00 \pm 0.38^{*}$ & $1.01 \pm 0.29$ \\
\hline \multirow[t]{2}{*}{ IL-17R } & Cornea & $1.00 \pm 0.32$ & $1.13 \pm 0.49$ & $0.51 \pm 0.20$ \\
\hline & Conjunctiva & $1.00 \pm 0.40$ & $1.92 \pm 0.24^{\star}$ & $0.82 \pm 0.18$ \\
\hline \multicolumn{5}{|c|}{ Th-17 pathway } \\
\hline \multirow[t]{2}{*}{ IL-17A } & Cornea & $1.00 \pm 0.55$ & $2.90 \pm 0.70^{*}$ & $1.89 \pm 0.66$ \\
\hline & Conjunctiva & $1.00 \pm 0.42$ & $2.58 \pm 0.51^{*}$ & $2.96 \pm 0.36^{* *}$ \\
\hline $\mathrm{ROR} \gamma \mathrm{t}$ & Cornea & $1.00 \pm 0.43$ & $1.20 \pm 0.48$ & $0.31 \pm 0.48$ \\
\hline \multirow[t]{2}{*}{ IL-17F } & Cornea & $1.00 \pm 0.43$ & $0.95 \pm 0.34$ & $0.47 \pm 0.50$ \\
\hline & Conjunctiva & $1.00 \pm 0.51$ & $1.40 \pm 0.55$ & $1.17 \pm 0.53$ \\
\hline \multirow[t]{2}{*}{ CCL20 } & Cornea & $1.00 \pm 0.52$ & $6.54 \pm 0.59^{\star *}$ & $2.52 \pm 0.44^{\star}$ \\
\hline & Conjunctiva & $1.00 \pm 0.38$ & $1.76 \pm 0.37$ & $0.53 \pm 0.41$ \\
\hline \multirow[t]{2}{*}{ STAT3 } & Cornea & $1.00 \pm 0.37$ & $0.96 \pm 0.29$ & $1.72 \pm 0.19$ \\
\hline & Conjunctiva & $1.00 \pm 0.36$ & $1.54 \pm 0.32$ & $1.37 \pm 0.37$ \\
\hline \multicolumn{5}{|c|}{ Th-1 pathway } \\
\hline \multirow[t]{2}{*}{$\mathrm{IFN}-\gamma$} & Cornea & $1.00 \pm 0.35$ & $3.31 \pm 0.52^{*}$ & $0.21 \pm 0.54$ \\
\hline & Conjunctiva & $1.00 \pm 0.35$ & $1.63 \pm 0.35$ & $2.27 \pm 0.41^{*}$ \\
\hline \multirow[t]{2}{*}{ IL-12 } & Cornea & $1.00 \pm 0.51$ & $1.38 \pm 0.30$ & $0.37 \pm 0.31$ \\
\hline & Conjunctiva & $1.00 \pm 0.32$ & $0.39 \pm 0.41$ & $0.38 \pm 0.61$ \\
\hline \multirow[t]{2}{*}{ IL-2 } & Cornea & $1.00 \pm 0.67$ & $4.34 \pm 0.63^{\star *}$ & $1.29 \pm 0.35$ \\
\hline & Conjunctiva & $1.00 \pm 0.34$ & $0.68 \pm 0.32$ & $0.72 \pm 0.48$ \\
\hline \multirow[t]{2}{*}{ IL-18 } & Cornea & $1.00 \pm 0.44$ & $1.01 \pm 0.38$ & $0.96 \pm 0.54$ \\
\hline & Conjunctiva & $1.00 \pm 0.45$ & $1.78 \pm 0.33$ & $1.08 \pm 0.33$ \\
\hline IL-18R & Cornea & $1.00 \pm 040$ & $0.86 \pm 0.36$ & $0.75 \pm 0.37$ \\
\hline
\end{tabular}




\begin{tabular}{|c|c|c|c|c|}
\hline Gene & Location & \multicolumn{3}{|c|}{ Relative fold increase } \\
\hline \multirow{2}{*}{ t-Bet } & Conjunctiva & $1.00 \pm 0.53$ & $1.20 \pm 0.32$ & $0.32 \pm 0.23$ \\
\hline & Conjunctiva & $1.00 \pm 0.56$ & $1.03 \pm 0.16$ & $0.63 \pm 0.18$ \\
\hline \multirow[t]{2}{*}{ IL-4 } & Cornea & ND & ND & ND \\
\hline & Conjunctiva & $1.00 \pm 0.36$ & $0.55 \pm 0.60$ & $0.96 \pm 0.28$ \\
\hline \multirow[t]{2}{*}{ IL-13 } & Cornea & $1.00 \pm 0.40$ & $1.26 \pm 0.30$ & $0.21 \pm 0.87$ \\
\hline & Conjunctiva & $1.00 \pm 0.37$ & $0.49 \pm 0.38$ & $0.67 \pm 0.46$ \\
\hline GATA-3 & Cornea & $1.00 \pm 0.56$ & $0.45 \pm 0.36$ & $0.35 \pm 0.40$ \\
\hline
\end{tabular}

IFN- $\gamma$, interferon-gamma; IL, interleukin; MMP-9, matrix metalloproteinase-9; ND, non-detectable; TGF, transforming growth factor; Th-17, T helper 17. Data are shown as the mean \pm s.d.; $n=8$ per time point, ${ }^{*} \mathrm{P}<0.05,{ }^{*} \mathrm{P}<0.01$ vs. C57BL/6 UT.
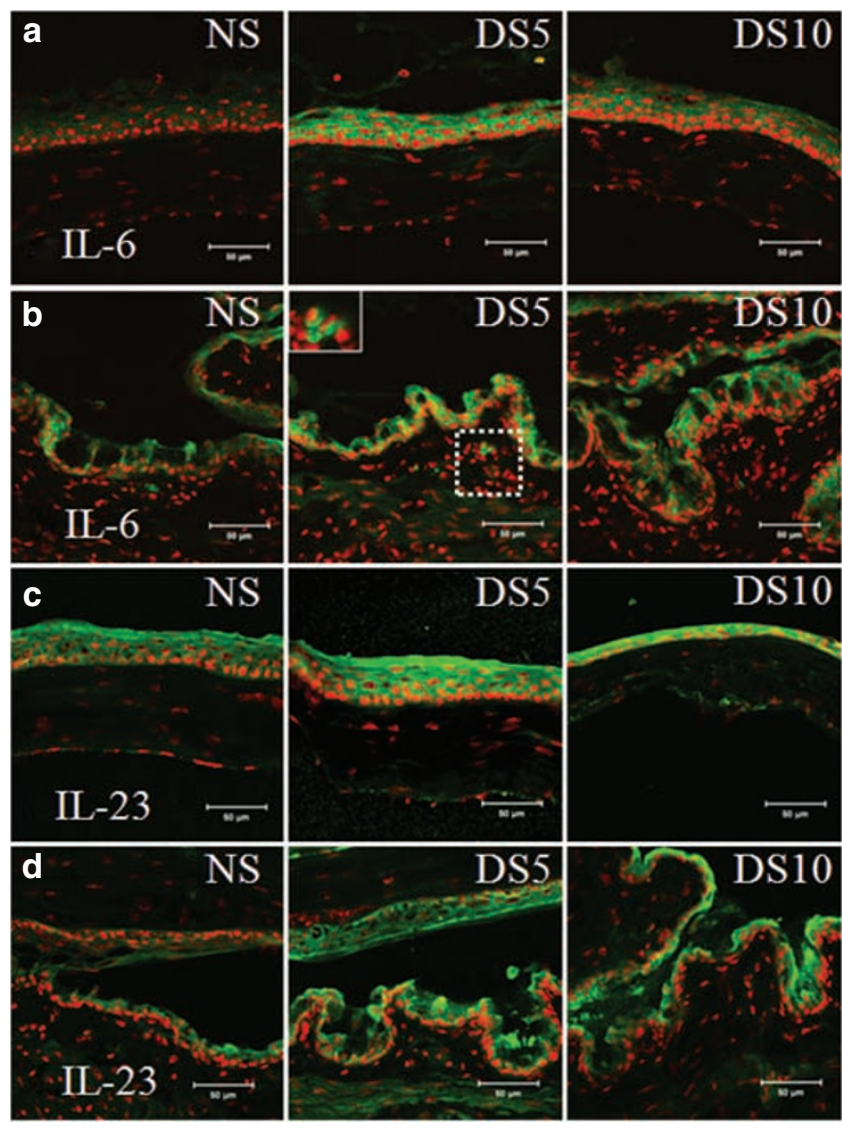
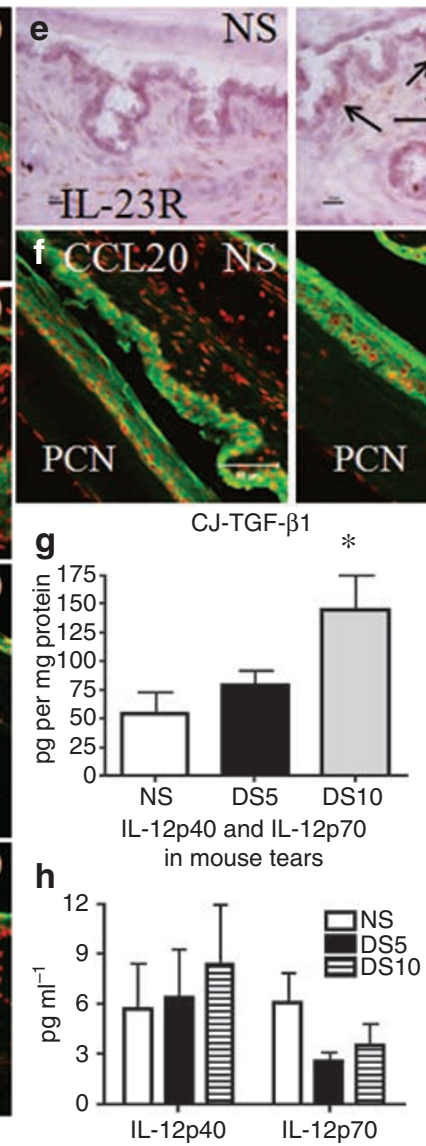
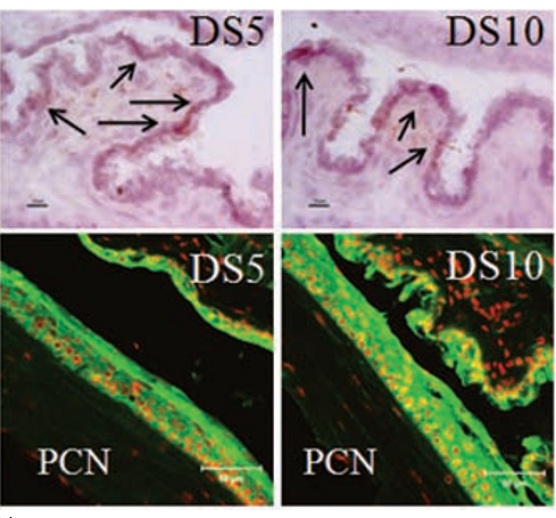

CJ-TGF- $\beta 2$
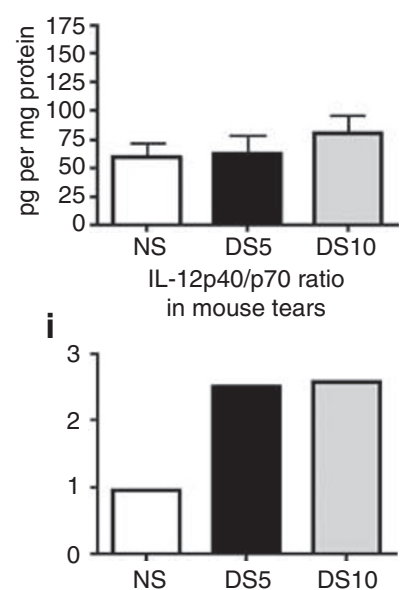

Figure 3 (a-f) Laser scanning immunofluorescent confocal microscopy of the cornea (a, c, f) and conjunctiva (b, d, e) sections stained for IL-6 ( $\mathbf{a}$ and $\mathbf{b}$ ) or IL-23 (c and d) and CCL20 (f) (in green), with propidium iodide nuclear counterstaining (in red) in NS control (NS), 5 days (DS5) or 10 days (DS10) of desiccating stress (DS) ( $n=5$ per group). (b) Small inset: higher magnification of an IL-6-positive cell in the stroma of the conjunctiva in the goblet-cell-rich area. Scale bar $=50 \mu \mathrm{m}$. (e) Immunohistochemical staining for IL-23R positively stained cells (red) indicated by arrows in conjunctival sections of NS, DS5, and DS10 mice ( $n=5$ per group). Scale bar $=25 \mu \mathrm{m}$. (g) Mean \pm S.d. of levels of TGF- $\beta 1$ and TGF- $\beta 2$ protein in conjunctival lysates obtained from NS control (NS), 5 days (DS5) or 10 days (DS10) of desiccating stress (DS). Data are presented as pg mg ${ }^{-1}$ of three independent experiments. (h) IL-12p40 and IL-12p70 concentration in tear fluid samples measured by immunobead assay obtained from mice subjected to DS. Data are presented as mean \pm s.d. of three independent experiments. (i) Ratio of both subunits. ${ }^{\star} P<0.05$ DS5 or DS10 vs. NS group. IL, interleukin; TGF, transforming growth factor. 

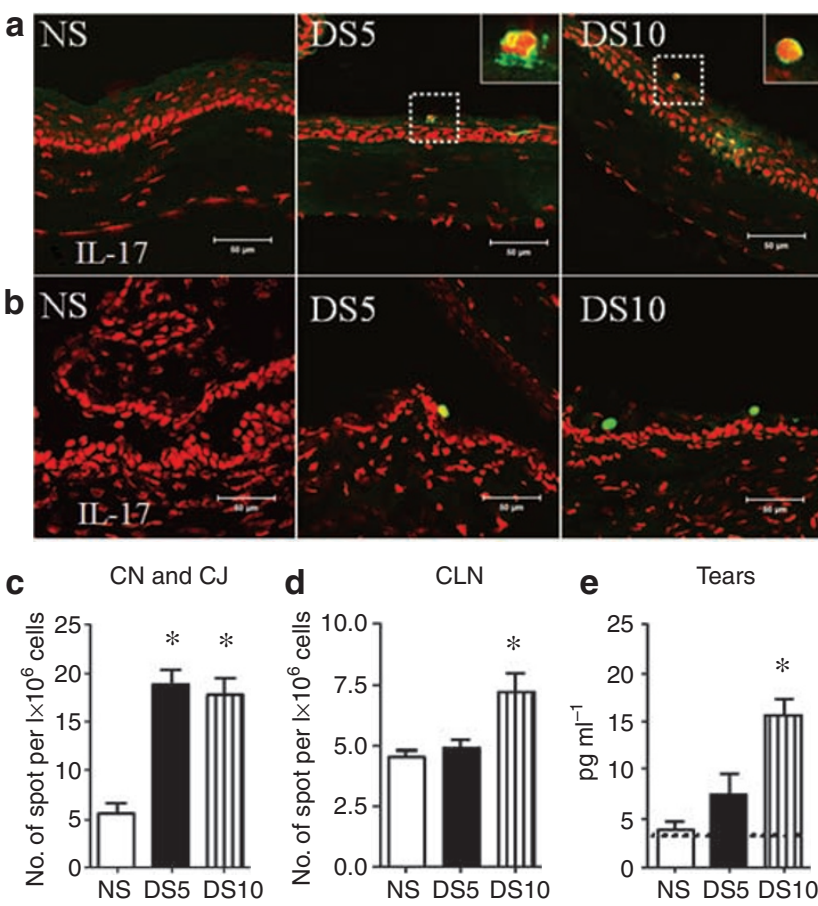

e

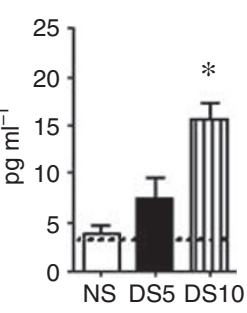

Figure 4 ( $\mathbf{a}$ and $\mathbf{b}$ ) Laser scanning immunofluorescent confocal microscopy of corneal (a) and conjunctival (b) sections stained for IL17 (in green) with propidium iodide nuclear counterstaining (in red) in NS control (NS), 5 days (DS5) or 10 days (DS10) of desiccating stress (DS) ( $n=5$ per group). Insets: higher magnification of IL-17-positive cells in DS5 and DS10 in mid-peripheral cornea. Scale bar $=50 \mu \mathrm{m}$. (c and d) Mean \pm s.d. of three independent IL-17 ELISPOTS, showing IL17-producing cells isolated from the cornea $(\mathrm{CN})$ and conjunctiva $(\mathrm{CJ}$, both in (c)) and cervical lymph nodes (CLN, in (d)) after desiccating stress. (e) IL-17 concentration in tear fluid samples obtained from mice subjected to DS measured by immunobead assay. The dotted line indicates the lower limit of detection in the assay. Data are presented as mean \pm s.d. of three independent experiments. CLN, cervical lymph node; $\mathrm{IL}$, interleukin.

These results indicate that the ocular surface is a TGF- $\beta-$, IL6-, and IL-23-rich environment. Desiccating stress was found to stimulate the production of CCL20 and IL-23R, factors that may facilitate the influx of IL-17-producing cells.

\section{IL-17-producing cells are visualized on the ocular surface after desiccating stress}

The expression of IL-17 on the ocular surface was evaluated in the cornea and conjunctiva by immunofluorescent staining and ELISPOT bioassay and in tear fluid by Luminex immunobead assay.

In contrast to the NS group, in which no cells were seen, DE stimulated an influx of IL-17-positive (+) cells in mid-peripheral cornea and in the goblet-cell-rich areas of the conjunctiva (DS5 and DS10) (Figure 4a and b). The influx of IL-17+ cells paralleled an increase in CCL20 expression in the cornea (Figure 3f).

To confirm IL-17 production after DS, we performed IL17 ELISPOT using freshly isolated cells from the cornea and conjunctiva and draining CLN. Our results, presented in Figure $4 c$, show a significant increase of IL-17-producing cells in the cornea and conjunctiva at DS5 and DS10, with a late increase (at DS10) of IL-17-producing cells in the CLN. We also observed a significant increase in the concentration of IL-17 in tears at DS10 (Figure 4e). To further evaluate if this Th-17-skewed deviation is unique to desiccating stress on the ocular surface, we evaluated the effects of another type of ocular surface insult by creating a unilateral sterile corneal epithelial defect in a separate group of mice. We isolated cells from the cornea and conjunctiva 5 days after the initial insult and we performed IL-17 ELISPOT. There was no increase in the number of IL-17-producing cells in either eye (data not shown), suggesting that increased IL-17 production is not a nonspecific response to ocular surface trauma.

These findings indicate that DS increases the number of IL17-producing cells in the ocular surface and prompted us to further investigate the role of IL-17 in DS.

\section{Neutralization of IL-17 in vivo ameliorates desiccating stress-induced corneal epithelial barrier dysfunction}

Dry eye and DS are well recognized to disrupt corneal epithelial barrier function. In humans, corneal permeability to fluorescein dye is currently used clinically to evaluate the severity of corneal epithelial barrier disruption. Corneal barrier disruption is associated with an increased risk of sterile and infectious corneal ulceration, corneal haze, and decreased vision.

To further evaluate the roles of IL-17 and IFN- $\gamma$ in the pathogenesis of the corneal epithelial disease that develops in response to DS, an in vivo IL-17 rat antibody neutralization was performed in separate groups of C57BL/ 6 and IFN- $\gamma \mathrm{KO}$ mice subjected to DS for 5 days (DS5 + anti-IL-17). Mice receiving rat IgG served as controls (DS5 + IC). We have reported earlier that desiccating stress induces an acute disruption of corneal barrier by 5 days in our mouse model; ${ }^{39}$ therefore, we evaluated the effects of IL-17 neutralization when disruption of barrier function is maximal.

A significant increase in corneal permeability to $70-\mathrm{kDa}$ Oregon Green Dextran-488 was found in DS5 compared with the NS group in both strains, although the magnitude of increase was significantly higher in the C57BL/6 strain (Figure 5a and d). Neutralization of IL-17 significantly decreased corneal permeability to Oregon Green Dextran-488 at DS5 in both strains, compared with DS5 + IC, which was no different than the DS5 group (Figure $\mathbf{5 a}$ and $\mathbf{d}$ ).

These results indicate that although both IL-17 and IFN- $\gamma$ may be involved in the corneal epithelial pathology in DE, IL-17 may be the dominant player in acute barrier dysfunction.

\section{IL-17 induces metalloproteinase production on the ocular surface and in human cultured epithelial cells}

We have reported earlier that increased MMP production disrupts corneal barrier function in response to experimental desiccating stress. ${ }^{36,40}$ Specifically, DS was found to increase MMP-9 and MMP-3, the latter being the main activator of MMP-9. As IL-17 has also been reported to upregulate MMPs, ${ }^{4,5}$ we evaluated if in vivo neutralization of IL-17 would have an effect on MMP levels. 

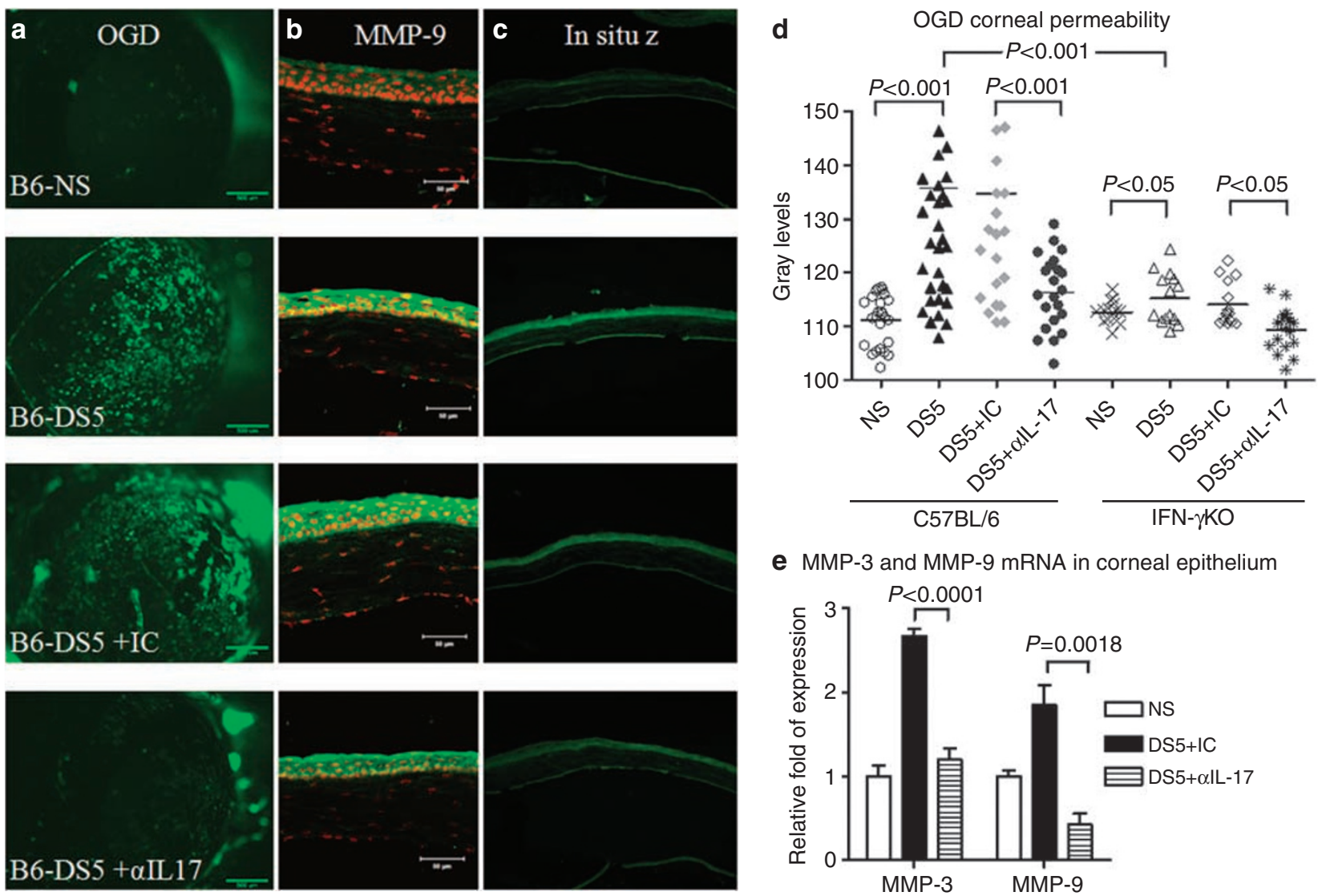

Figure 5 (a) Representative digital images in C57BL/6 (B6) mice (in (a)) used to score Oregon Green Dextran-488 (OGD) permeability (in d) among the treatment groups (NS=non-stressed controls, DS5=desiccating stress for 5 days, DS5+ $1 \mathrm{lL}-17=\mathrm{DS} 5$ treated with anti-IL-17 antibody, DS5 + IC = DS5 treated with rat antibody isotype control). (b) Laser scanning immunofluorescent confocal microscopy of cornea sections stained for MMP-9 (in green) with propidium iodide nuclear counterstaining (in red) among the treatment groups described above ( $n=5$ per group). (c) In situ zymography (in situ z) of central corneal sections of NS, DS5, DS5+alL-17, and DS5+IC mice. The fluorescence intensity is proportional to gelatinase activity within the tissue. (d) Mean \pm s.d. of corneal OGD score (in gray levels) measured by the Metavue Software by two masked observers among the treatment groups in C57BL/6 and IFN- $\gamma$ knockout mice (IFN- $\gamma \mathrm{KO}$ ) ( $n=30$ eyes per group). (e) MMP-3 and MMP-9 mRNA transcript levels in the corneal epithelium of C57BL/6 mice subjected to desiccating stress after treatment with either anti-IL-17 antibody or isotype control. IL, interleukin; MMP-9, matrix metalloproteinase-9.

We performed immunostaining for MMP-9 in corneal tissue sections prepared from NS, DS5, DS5 + anti-IL-17, and DS + IC (Figure 5b). Desiccating stress markedly increased MMP-9 immunoreactivity in all layers of the cornea. Neutralization of IL-17 partially decreased the staining intensity as opposed to the isotype control group that had a similar appearance to DS5. We also used in situ zymography to assay all gelatinase activity in the serial tissue sections. In this activity assay, sections are incubated with a fluorescent-conjugated gelatin. The fluorescence intensity in the tissue is proportional to the enzyme activity, because cleavage of the fluorescent substrate yields visible fluorescence. As shown in Figure 5c, we observed a marked increase in fluorescence in DS5 and DS5 + IC, whereas neutralization of IL-17 was found to decrease the DS-induced gelatinolytic activity in the corneal epithelium.

To confirm this finding, we evaluated levels of MMP-9 and MMP-3 transcripts in the corneal epithelia of animals that received the in vivo neutralization of IL-17. Neutralization of IL-17 significantly reduced levels of MMP-9 and MMP-3 transcripts in these cells (Figure 5e), but no significant change in the levels of IL- 6 , TGF- $\beta 1$, and IFN- $\gamma$ mRNA transcripts was observed (data not shown).

We investigated the effects of exogenous administration of IL-17 on MMP production by primary cultured human corneal epithelial cells as well as barrier function to sodium fluorescein dye in a corneal epithelial cell line. Treatment of corneal epithelial cells with IL-17A for $16 \mathrm{~h}$ significantly increased the levels of MMP-3 (4.65 \pm 0.81 -fold increase, $P=0.04$ ) and MMP-9 (2.88 \pm 0.37 -fold increase, $P=0.03)$ transcripts. Treatment of the corneal epithelial cell line with IL-17A for 5 days induced a 30\% increase in permeability to sodium fluorescein compared with untreated controls (data not shown).

\section{DISCUSSION}

Our studies evaluated human and experimental murine DE for evidence of Th-17 inflammation. In humans, we evaluated the expression of MMP-9, inflammatory cytokines, and Th-1-, Th2 -, and Th-17-associated genes and we found increased levels of MMP-9, IL-1 $\beta$, TNF- $\alpha$, IL-17, and its inducers, as well as IFN- $\gamma$, in the conjunctiva from DE patients. There was a significant 
positive correlation between levels of TGF- $\beta 1$, IL- 23 , and IL- 6 mRNA and clinical severity of DE parameters. These results were corroborated in the mouse model.

Increased production of IL- 6 on the ocular surface has been identified in human DE patients in a number of studies. ${ }^{41,42}$ We found that IL- 6 was the only cytokine in $\mathrm{DE}$ tears with a concentration 2 s.d. above normal levels. We also found that IL- 6 tear concentrations showed strong correlation with the severity of ocular surface epithelial disease. $^{43}$

Experimental desiccating stress to the mouse ocular surface was found to increase the number of $\mathrm{CD} 4+\mathrm{T}$ cells in the conjunctival epithelium and stimulate the production of Th17 -associated genes, as well as IFN- $\gamma$. Desiccating stress also increased the number of IL-17-producing cells in the cornea and conjunctiva and increased the IL-17 concentration in tears. Antibody neutralization of IL-17 in C57BL/ 6 wild-type and in IFN- $\gamma \mathrm{KO}$ mice ameliorated desiccating stress-induced corneal epithelial barrier dysfunction, and it was accompanied by a decrease in MMP-3 and -9 gene expression, MMP-9 protein expression, and gelatinolytic activity in the corneal epithelium. Taken together, these findings indicate that Th-17 has a role in the corneal epithelial pathology that develops in DE.

The ocular surface is a TGF- $\beta 1$ - and IL-6-rich environment. ${ }^{41,44}$ These two cytokines are recognized to be essential in the induction of Th- 17 cells. ${ }^{10-12}$ TGF- $\beta$ synergizes with IL- 6 to induce the expression of the transcription factor ROR $\gamma \mathrm{t}$, a key regulator of Th-17 differentiation. ${ }^{10-13}$ We found a progressive increase in the number of $\mathrm{CD} 4+\mathrm{T}$ in the conjunctival epithelium over 10 days of DS. As the local cytokine milieu is very important in the maturation and differentiation of naive $\mathrm{T}$ cells, we hypothesized that the ocular surface in DE promotes Th-17 inflammation.

Our findings of increased Th-17 and Th-1 (IFN- $\gamma$ ) cytokines in the conjunctiva obtained from human DE patients prompted us to further investigate the response of the ocular surface and CLN to desiccating stress in mice. Similar to humans, we found that experimental DE induced a significant increase of MMP-9-, IFN- $\gamma$-, and Th-17-associated cytokines. Dry eye significantly stimulated the production of IL-23, IL-6, and TGF- $\beta$, the three factors that are important in driving differentiation and survival of Th-17 cells, in the cornea and conjunctiva. ${ }^{10-12,14,15}$ Furthermore, it stimulated the production of MMP-9, which is a recognized activator of latent TGF- $\beta$. Interestingly, the cornea and conjunctiva had more significant changes in Th-17-related gene expression than the draining lymph nodes. It is of particular interest that we found an increased expression of IL-17R and IL-23R in the conjunctiva, with IL-23R + cells noted to infiltrate the conjunctival epithelium. IL-23R is considered as one of the hallmarks of Th-17 differentiation and is not expressed by naive cells. ${ }^{45}$ We found an increased expression of IL-17A in (tears, cornea, and conjunctiva) and the lineage-specific transcription factor ROR $\gamma \mathrm{t}$ in the conjunctiva. We also found a higher number of IL-17-producing cells in the cornea and conjunctiva than in the CLN population. Our findings of higher levels of expression of Th-17-associated cytokines and IFN- $\gamma$ in our mouse model are consistent with earlier findings in humans. Increased levels of IL-17 and IL-23 were found earlier in tears, saliva, and in salivary gland biopsies obtained from Sjögren's patients. ${ }^{46,47}$ Elevated concentrations of IL- 6 were also found in sera and saliva obtained from Sjögren's Syndrome patients. ${ }^{46}$ Taken together, these findings suggest that the ocular surface environment offers an enabling cytokine milieu for the differentiation of Th-17 cells in response to desiccating stress.

CCL20 is a potent chemoattractant that drives Th-17+ cells to sites of inflammation. CCL20 is stimulated by IL-17A itself $^{2}$ and by IL- 1 and TNF- $\alpha,{ }^{48}$ the two cytokines that are highly expressed by the cornea epithelium in DE. ${ }^{32,33}$ We found a sixfold increase in levels of CCL20 mRNA transcripts in the cornea at DS5, compared with controls; these results were confirmed by immunostaining. This increase of CCL20 may facilitate the influx of IL-17 + cells that we observed in the mid-peripheral cornea, which is devoid of $\mathrm{T}$ cells under normal circumstances. CCL20 and its ligand, CCR6, were found to be highly expressed in psoriatic lesions, ${ }^{2}$ another epithelial disease in which Th-17 is implicated. ${ }^{22}$

In addition to increased IL-17 production, we found an increase in IFN- $\gamma$ that peaked in the cornea at day 5 and in the conjunctiva at day 10. It has become apparent in a number of other models of autoimmunity, such as experimental autoimmune encephalomyelitis and autoimmune uveitis, that increased expressions of IL-17 and IFN- $\gamma$ are not mutually exclusive. The relative contribution of IL-17 and IFN- $\gamma$ to the pathological changes in these models remains controversial. For example, in experimental autoimmune uveitis induced by immunization with intra-retinal binding protein and complete Freud's adjuvant, treatment with IL-17A-neutralizing antibody reduced disease severity, ${ }^{26}$ whereas neutralization of IFN- $\gamma$ exacerbated disease $^{49}$ and treatment with recombinant IFN- $\gamma$ was shown to protect against disease..$^{50}$ In contrast, experimental autoimmune uveitis induced by immunizing mice with intra-retinal binding protein-pulsed dendritic cells is characterized by an influx of T cells producing large amounts of IFN- $\gamma$, but little IL-17. ${ }^{26}$ IFN- $\gamma$-deficient mice treated with this protocol did not develop disease. The discrepancies noted between these studies may be explained by the use of complete Freud's adjuvant in the immunization model, which may exaggerate the importance of IL-17. Increased production of IL-17 in our study cannot be ascribed to adjuvants because they are not utilized in our environmental stress model. Increased levels of IFN- $\gamma$ and IL17 were found in human samples and in our mouse model. It is possible that IL-17 and IFN- $\gamma$ each has unique functions on the ocular surface in response to desiccating stress. IL-17, in conjunction with IL- 1 and TNF- $\alpha$, appears to stimulate the production of MMPs by the corneal epithelium, which mediates proteolytic disruption of epithelial tight junctions that maintain corneal barrier function. In contrast, IFN- $\gamma$ inhibits production of MMPs by certain tissues, and thus, it may antagonize the effects of IL-17. Furthermore, IFN- $\gamma$ stimulates the production of cornified envelope precursors by the ocular surface epithelia, ${ }^{38}$ which create more of a skin-like barrier in these mucosal epithelia. 
We have reported earlier that IFN- $\gamma$ contributed to conjunctiva goblet cell loss in our mouse model ${ }^{38}$ however, we did not evaluate the effects of IFN- $\gamma$ on corneal permeability at that time. Our current experiments indicate that loss of IFN $-\gamma$ decreases the severity of corneal barrier disruption; however, neutralization of IL-17 significantly improved barrier function in both C57BL/6 and IFN- $\gamma$ KO mice. These results indicate that both Th- 1 and Th-17 pathways may be involved in ocular surface response to DS, with IL-17 having the dominant role in the cornea.

These findings have great clinical implications, because increased corneal permeability to fluorescein dye is a hallmark of DE, and it is associated with increased corneal epithelial desquamation (punctate epithelial erosions) and corneal surface irregularity, changes that result in decreased functional vision. We have shown earlier that MMP-9 produced and activated in response to desiccating stress disrupts corneal barrier function and observed that MMP-9-deficient mice were resistant to barrier disruption in desiccating stress. ${ }^{40}$ As IL-17 has been reported to upregulate the expression of MMPs, ${ }^{4,5}$ we evaluated if IL-17 neutralization would decrease expression of MMP-9, as well as MMP-3 (a potent physiologic activator of MMP-9). We found a significant decrease in both MMP-9 and MMP-3 transcripts in mice receiving IL-17-neutralizing antibody. This was accompanied by decreased MMP-9 immunoreactivity and gelatinolytic activity in the cornea. As further evidence, a marked increase in both MMP-9 and MMP-3 was found in cultured human corneal epithelial cells treated with recombinant IL-17 and a corresponding $30 \%$ increased permeability to sodium fluorescein was observed.

In summary, we showed that desiccating stress elicited a Th- 1 and Th-17 response on the ocular surface and that IL-17 is involved in the disruption of corneal barrier function, which is the most sight-threatening complication of DE. The ocular surface has high levels of Th-17-inducing factors, such as IL-6, IL-23, and TGF- $\beta$, that appear to render it a predisposed site for promoting Th-17 differentiation and survival.

\section{METHODS}

Real-time PCR. The RNA concentration was measured by its absorption at $260 \mathrm{~nm}$ and samples were stored at $-80^{\circ} \mathrm{C}$ until use. Firststrand cDNA was synthesized with random hexamers by M-MuLV reverse transcription (Ready-To-Go You-Prime First-Strand Beads; GE Healthcare, Arlington Heights, NJ), as described earlier. ${ }^{35}$

Real-time PCR was performed with specific MGB probes (Taqman; Applied Biosystems (ABI), Foster City, CA) and PCR master mix (Taqman Gene Expression Master Mix), in a commercial thermocycling system (Mx3005P QPCR System; Stratagene, La Jolla, CA), according to the manufacturer's recommendations. Human probes used were GAPDH, MMP-9, IL-6, IL-23, TGF- $\beta 1$, TGF- $\beta 2$, IL-17A, IFN- $\gamma$, IL-2, IL-12, IL-4, and IL-13 (assay IDs: Hs99999905, Hs00234579, Hs00174131, Hs00372324, Hs99999918, Hs00234244, Hs00174383, Hs999904, Hs00174114, Hs00188405, Hs00174122, and Hs00174379, respectively), and murine MGB probes used were GAPDH, IL-6, IL23 , TGF- $\beta 1$, TGF- $\beta 2$, IL-17A, IL-23 receptor, IL- 17 receptor, ROR $\gamma \mathrm{t}$, STAT3, IL-21, IL-22, CCL20, IL-17F, IFN- $\gamma$, IL-2, IL-12, T-bet, IL-4, IL-13, GATA-3, IL-18, IL-18R, STAT3, STAT6, IL-21, and IL-22 (assay IDs: Mm99999915, Mm00446490, Mm00518984, Mm004417241, Mm00436952, Mn00439619, Mm00519942, Mm00434214, Mm00441139, Mm00456961, Mm00517640, Mm00444241,
Mm00444228, Mm00521423, Mm00801778, Mm00434256, Mm00434165, Mm00450960, Mm00445259, Mm00434204, Mm00484683, Mm00434225, Mm00515180, and Mm01160477, respectively). The GAPDH gene was used as an endogenous reference for each reaction. The results of quantitative PCR were analyzed by the comparative $C_{\mathrm{t}}$ method in which the target change $=2^{-} \Delta \Delta^{C \mathrm{t}}$ The results were normalized by the $C_{\mathrm{t}}$ value of GAPDH and the mean $C_{\mathrm{t}}$ of relative mRNA level in the normal control group (normal subjects and NS mice) was used as the calibrator.

Mice. C57BL/ 6 and IFN- $\gamma$ knockout mice (in C57BL/ 6 background, B6.129S7-Ifng ${ }^{t m I T s} / J$, IFN- $\gamma \mathrm{KO}$ ) from both genders, 6-8 weeks old, were purchased from The Jackson Laboratory (Bar Harbor, ME). All animal experiments were approved by the institutional animal care and use committees at Allergan and Baylor College of Medicine. All studies adhered to the Association for Research in Vision and Ophthalmology Statement for the Use of Animals in Ophthalmic and Vision Research. All experiments were performed in C57BL/6 mice unless noted.

Induction of DS in mice. Desiccating stress was induced by subcutaneous injection of scopolamine hydrobromide $(0.5 \mathrm{mg}$ per $0.2 \mathrm{ml}$; SigmaAldrich, St Louis, MO) four times a day $(0800,1200,1400$, and 1700 hours), alternating flanks of mice, as described earlier. ${ }^{34-37}$ Mice were placed in a cage with a perforated plastic screen on one side to allow airflow from a fan placed 6 inches in front of it for $16 \mathrm{~h}$ per day. Room humidity was maintained at 30-35\%. DS was induced for either 5 or 10 consecutive days (DS5 or DS10, respectively). This model of DE disease has been used earlier with no discernible ill effects from the scopolamine treatment or low humidity. ${ }^{37}$ Control mice were maintained in an NS environment containing $50-75 \%$ relative humidity without exposure to forced air.

Rat anti-mouse IL-17 serum. A peptide, CSSRRPSDYLNRSTSPW TLHRNEDPDR, corresponding to mouse IL-17 (aa62-aa89) was synthesized based on an amino-acid sequence that provided optimal antigenicity and hydrophilicity (UT Southwestern Protein Chemistry Technology Center). Rats were immunized intrasplenically with $100 \mu \mathrm{g}$ peptide conjugated with Keyhole limpet hemocyanin $(\mathrm{KLH})+20 \mu \mathrm{g}$ CpG DNA, followed, 1 month later, by one subcutaneous injection of $100 \mu$ g peptide conjugated with $\mathrm{KLH}+20 \mu \mathrm{g}$ CpG DNA + complete Freund's adjuvant. Rats were then injected subcutaneously with a boosting cocktail $(100 \mu \mathrm{g}$ peptide conjugated with $\mathrm{KLH}+20 \mu \mathrm{g} \mathrm{CpG}$ DNA + incomplete Freund's adjuvant) every 2 weeks for 2 months. Rat serum was collected and tested for polyclonal anti-mouse IL-17 by enzyme-linked immunosorbent assay screening with the synthetic peptide and by western blot analysis using recombinant mouse IL-17A (eBiosciences, San Diego, CA) (data not shown).

In vivo depletion of IL-17 in C57BL/6 and IFN- $\gamma$ KO mice. Groups of C57BL/6 ( $n=40$ per group) and IFN- $\gamma$ KO mice ( $n=10$ per group) received intraperitoneal injections of either a rat anti-IL-17-neutralizing polyclonal serum (DS5 + anti-IL-17, $500 \mu \mathrm{g}$ per mice) or rat IgG (DS5 + isotype control (IC), $500 \mu \mathrm{g}$ per mice; Sigma-Aldrich). The injection regimen was as follows: day -4 , day -2 , day 0 , day +2 , and day +4 . Both groups were subjected to DS (at day 0 ) as described above and they were euthanized on day 5 of desiccating stress.

Isolation of murine cells. The eyes and lids of mice $(n=5$ per experiment, in four independent sets of experiments, total of 20 per group, in NS, DS5, and DS10 groups and $n=5$ per experiment, in two independent sets of experiments in corneal scar and control groups) were excised, pooled, and incubated in $10 \mathrm{ml}$ of $5 \mathrm{mg} \mathrm{ml}^{-1}$ Dispase II (Roche Molecular Biochemicals, Indianapolis, IN) in a shaker at $37^{\circ} \mathrm{C}$ for $1 \mathrm{~h}$, followed by neutralization with Hank's Buffered Salt Solution (Invitrogen-Gibco, Grand Island, NY) supplemented with 3\% fetal bovine serum (Hyclone, Logan, UT). The bulbar and tarsal conjunctivae were scraped with cytology brushes under a dissecting microscope. Respective superficial 
CLNs were surgically excised, smashed in between two sterile frosted glass slides, and made into a single-cell suspension. Cell populations were individually collected, centrifuged at 2000 r.p.m. $\times 5$ min, filtered, and resuspended. Cells collected by this technique were used either for flow cytometry (desiccating stress experiment) or for ELISPOT (desiccating stress and corneal ulcer experiment).

Mouse IL-17 ELISPOT. Replicate $50-\mu$ l cell suspensions containing $1.0 \times 10^{6}$ freshly isolated cells (as described above) were added to 96-well polyvinylidene fluoride plates (Milipore, Billerica, MA) and precoated with anti-mouse IL-17 capture antibody (R\&D Systems, Minneapolis, MN). Wells containing either cells or positive control (3 ng per well of recombinant mouse IL-17A; R\&D Systems) or media alone (negative control) were incubated at $37^{\circ} \mathrm{C}$ with $\mathrm{CO}_{2}$ for $24 \mathrm{~h}$ in RPMI media (Invitrogen-Gibco). After washing, the plate was incubated overnight at $4{ }^{\circ} \mathrm{C}$, with biotinylated goat antimouse IL-17 detection antibody (R\&D Systems), followed by incubation with streptavidin-horseradish peroxidase (R\&D Systems) on the next day, for $2 \mathrm{~h}$. Red color development was achieved by incubating NovaRed peroxidase substrate (Vector Laboratories, Burlingame, CA) for $15 \mathrm{~min}$. The polyvinylidene fluoride membrane was dried and the individual wells were punched out from the plate. The positive red spots were counted under a dissecting microscope (SMZ 1500; Melville, NY). Replicate wells were averaged from three individual experiments. Results are presented as number of spots per $1 \times 10^{6}$ cells.

SUPPLEMENTARY MATERIAL is linked to the online version of the paper at http://www.nature.com/mi

\section{ACKNOWLEDGMENTS}

This study was funded by NIH grants EY11915 (SCP), RPB, Oshman Foundation, William Stamps Farish Fund, Hamill Foundation, and Allergan.

\section{DISCLOSURE}

K.F. Siemasko and M.E. Stern are employees of Allergan.

() 2009 Society for Mucosal Immunology

\section{REFERENCES}

1. Bettelli, E., Oukka, M. \& Kuchroo, V.K. T(H)-17 cells in the circle of immunity and autoimmunity. Nat. Immunol. 8, 345-350 (2007).

2. Homey, B. et al. Up-regulation of macrophage inflammatory protein-3 alpha/CCL20 and CC chemokine receptor 6 in psoriasis. J. Immunol. 164, 6621-6632 (2000).

3. Liang, S.C., Tan, X.Y., Luxenberg, D.P., Dunussi-Joannopoulos, K., Collins, M. \& Fouser, L.A. Interleukin (IL)-22 and IL-17 are coexpressed by Th17 cells and cooperatively enhance expression of antimicrobial peptides. J. Exp. Med. 203, 2271-2279 (2006).

4. Cortez, D.M. et al. IL-17 stimulates MMP-1 expression in primary human cardiac fibroblasts via p38. Am. J. Physiol. Heart Circ. Physiol. 293, H3356-H3365 (2007).

5. Yagi, Y., Andoh, A., Inatomi, O., Tsujikawa, T. \& Fujiyama, Y. Inflammatory responses induced by interleukin-17 family members in human colonic subepithelial myofibroblasts. J. Gastroenterol. 42, 746-753 (2007).

6. Jones, C.E. \& Chan, K. Interleukin-17 stimulates the expression of interleukin-8, growth-related oncogene-alpha, and granulocyte-colonystimulating factor by human airway epithelial cells. Am. J. Respir. Cell Mol. Biol. 26, 748-753 (2002).

7. Happel, K.I. et al. Divergent roles of IL-23 and IL-12 in host defense against Klebsiella pneumoniae. J. Exp. Med. 202, 761-769 (2005).

8. Chung, D.R. et al. CD4+ T cells mediate abscess formation in intraabdominal sepsis by an IL-17-dependent mechanism. J. Immunol. 170, 1958-1963 (2003).

9. Huang, W., Na, L., Fidel, P.L. \& Schwarzenberger, P. Requirement of interleukin-17A for systemic anti-Candida albicans host defense in mice. J. Infect. Dis. 190, 624-631 (2004).
10. Veldhoen, M., Hocking, R.J., Atkins, C.J., Locksley, R.M. \& Stockinger, B. TGFbeta in the context of an inflammatory cytokine milieu supports de novo differentiation of IL-17-producing T cells. Immunity 24, 179-189 (2006).

11. Bettelli, E. et al. Reciprocal developmental pathways for the generation of pathogenic effector TH17 and regulatory T cells. Nature 441, 235-238 (2006).

12. Mangan, P.R. et al. Transforming growth factor-beta induces development of the $\mathrm{T}(\mathrm{H}) 17$ lineage. Nature 441, 231-234 (2006).

13. Ivanov, I.I. et al. The orphan nuclear receptor RORgammat directs the differentiation program of proinflammatory IL-17+ Thelper cells. Cell 126, $1121-1133$ (2006)

14. Yen, D. et al. IL-23 is essential for T cell-mediated colitis and promotes inflammation via IL-17 and IL-6. J. Clin. Invest. 116, 1310-1316 (2006).

15. Langowski, J.L. et al. IL-23 promotes tumour incidence and growth. Nature 442, 461-465 (2006).

16. costa-Rodriguez, E.V., Napolitani, G., Lanzavecchia, A. \& Sallusto, F. Interleukins 1 beta and 6 but not transforming growth factor-beta are essential for the differentiation of interleukin 17-producing human Thelper cells. Nat. Immunol. 8, 942-949 (2007)

17. Wilson, N.J. et al. Development, cytokine profile and function of human interleukin 17-producing helper T cells. Nat. Immunol. 8, 950-957 (2007).

18. Komiyama, Y. et al. IL-17 plays an important role in the development of experimental autoimmune encephalomyelitis. J. Immunol. 177, 566-573 (2006).

19. Nakae, S. et al. Antigen-specific T cell sensitization is impaired in IL-17deficient mice, causing suppression of allergic cellular and humoral responses. Immunity 17, 375-387 (2002).

20. Nakae, S., Nambu, A., Sudo, K. \& Iwakura, Y. Suppression of immune induction of collagen-induced arthritis in IL-17-deficient mice. J. Immunol. 171, 6173-6177 (2003).

21. Park, $H$. et al. A distinct lineage of $C D 4 T$ cells regulates tissue inflammation by producing interleukin 17. Nat. Immunol. 6, 1133-1141 (2005).

22. Haider, A.S. et al. Identification of cellular pathways of 'type 1,' Th17 T cells, and TNF- and inducible nitric oxide synthase-producing dendritic cells in autoimmune inflammation through pharmacogenomic study of cyclosporine A in psoriasis. J. Immunol. 180, 1913-1920 (2008).

23. Zaba, L.C. et al. Amelioration of epidermal hyperplasia by TNF inhibition is associated with reduced Th17 responses. J. Exp. Med. 204, 3183-3194 (2007).

24. Hwang, S.Y. \& Kim, H.Y. Expression of IL-17 homologs and their receptors in the synovial cells of rheumatoid arthritis patients. Mol. Cells 19, 180-184 (2005).

25. Chi, W. et al. Upregulated IL-23 and IL-17 in Behcet patients with active uveitis. Invest. Ophthalmol. Vis. Sci. 49, 3058-3064 (2008).

26. Luger, D. et al. Either a Th17 or a Th1 effector response can drive autoimmunity: conditions of disease induction affect dominant effector category. J. Exp. Med. 205, 799-810 (2008).

27. Moss, S.E., Klein, R. \& Klein, B.E. Incidence of dry eye in an older population. Arch. Ophthalmol. 122, 369-373 (2004).

28. Schein, O.D., Munoz, B., Tielsch, J.M., Bandeen-Roche, K. \& West, S. Prevalence of dry eye among the elderly. Am. J. Ophthalmol. 124, 723728 (1997).

29. Bandeen-Roche, K., Munoz, B., Tielsch, J.M., West, S.K. \& Schein, O.D. Self-reported assessment of dry eye in a population-based setting. Invest. Ophthalmol. Vis. Sci. 38, 2469-2475 (1997).

30. Bjerrum, K.B. Keratoconjunctivitis sicca and primary Sjogren's syndrome in a Danish population aged 30-60 years. Acta. Ophthalmol. Scand. 75, 281-286 (1997).

31. Miljanovic, B., Dana, R., Sullivan, D.A. \& Schaumberg, D.A. Impact of dry eye syndrome on vision-related quality of life. Am. J. Ophthalmol. 143, 409-415 (2007).

32. Pflugfelder, S.C., Jones, D., Ji, Z., Afonso, A. \& Monroy, D. Altered cytokine balance in the tear fluid and conjunctiva of patients with Sjogren's syndrome keratoconjunctivitis sicca. Curr. Eye Res. 19, 201211 (1999)

33. Solomon, A., Dursun, D., Liu, Z., Xie, Y., Macri, A. \& Pflugfelder, S.C. Proand anti-inflammatory forms of interleukin-1 in the tear fluid and conjunctiva of patients with dry-eye disease. Invest. Ophthalmol. Vis. Sci. 42, 2283-2292 (2001). 
34. Niederkorn, J.Y. et al. Desiccating stress induces T cell-mediated Sjogren's syndrome-like lacrimal keratoconjunctivitis. J. Immunol. 176, 3950-3957 (2006).

35. de Paiva, C.S. et al. Apical corneal barrier disruption in experimental murine dry eye is abrogated by methylprednisolone and doxycycline. Invest. Ophthalmol. Vis. Sci. 47, 2847-2856 (2006).

36. de Paiva, C.S. et al. Corticosteroid and doxycycline suppress MMP-9 and inflammatory cytokine expression, MAPK activation in the corneal epithelium in experimental dry eye. Exp. Eye Res. 83, 526-535 (2006).

37. Luo, L., Li, D.Q., Doshi, A., Farley, W., Corrales, R.M. \& Pflugfelder, S.C. Experimental dry eye stimulates production of inflammatory cytokines and MMP-9 and activates MAPK signaling pathways on the ocular surface. Invest. Ophthalmol. Vis. Sci. 45, 4293-4301 (2004).

38. de Paiva, C.S. et al. Dry eye-induced conjunctival epithelial squamous metaplasia is modulated by interferon-\{gamma\}. Invest. Ophthalmol. Vis. Sci. 48, 2553-2560 (2007).

39. Corrales, R.M., Villarreal, A., Farley, W., Stern, M.E., Li, D.Q. \& Pflugfelder, S.C. Strain-related cytokine profiles on the murine ocular surface in response to desiccating stress. Cornea 26, 579-584 (2007).

40. Pflugfelder, S.C. et al. Matrix metalloproteinase- 9 knockout confers resistance to corneal epithelial barrier disruption in experimental dry eye. Am. J. Pathol. 166, 61-71 (2005).

41. Yoon, K.C., Jeong, I.Y., Park, Y.G. \& Yang, S.Y. Interleukin-6 and tumor necrosis factor-alpha levels in tears of patients with dry eye syndrome. Cornea 26, 431-437 (2007).

42. Tishler, M., Yaron, I., Geyer, O., Shirazi, I., Naftaliev, E. \& Yaron, M. Elevated tear interleukin-6 levels in patients with Sjogren syndrome. Ophthalmology 105, 2327-2329 (1998)
43. Lam, H., Blieden, L., de Paiva, C.S., Farley, W.J., Stern, M.E. \& Pflugfelder, S.C. Tear cytokine profiles in dysfunctional tear syndrome. Am. J. Ophthalmol. 147, 198-205 (2009).

44. Pasquale, L.R., Dorman-Pease, M.E., Lutty, G.A., Quigley, H.A. \& Jampel, H.D. Immunolocalization of TGF-beta 1, TGF-beta 2, and TGF-beta 3 in the anterior segment of the human eye. Invest. Ophthalmol. Vis. Sci. 34, 23-30 (1993).

45. Parham, C. et al. A receptor for the heterodimeric cytokine IL-23 is composed of IL-12Rbeta1 and a novel cytokine receptor subunit, IL-23R. J. Immunol. 168, 5699-5708 (2002).

46. Nguyen, C.Q., Hu, M.H., Li, Y., Stewart, C. \& Peck, A.B. Salivary gland tissue expression of interleukin-23 and interleukin-17 in Sjogren's syndrome: findings in humans and mice. Arthritis Rheum. 58, 734-743 (2008).

47. Sakai, A., Sugawara, Y., Kuroishi, T., Sasano, T. \& Sugawara, S. Identification of IL-18 and Th17 cells in salivary glands of patients with Sjogren's syndrome, and amplification of IL-17-mediated secretion of inflammatory cytokines from salivary gland cells by IL-18. J. Immunol. 181, 2898-2906 (2008).

48. Shirane, J. et al. Corneal epithelial cells and stromal keratocytes efficiently produce CC chemokine-ligand 20 (CCL20) and attract cells expressing its receptor CCR6 in mouse herpetic stromal keratitis. Curr. Eye Res. 28, 297-306 (2004).

49. Billiau, A. et al. Enhancement of experimental allergic encephalomyelitis in mice by antibodies against IFN-gamma. J. Immunol. 140, 1506-1510 (1988).

50. Caspi, R.R. et al. Endogenous systemic IFN-gamma has a protective role against ocular autoimmunity in mice. J. Immunol. 152, 890-899 (1994). 